\title{
Expliciter l'implicite : à propos des « structures implicites d'organisation »
}

\section{Blandine Vanderlinden}

\section{(2) OpenEdition}

1 Journals

Édition électronique

URL : http://journals.openedition.org/communicationorganisation/2733

DOI : 10.4000/communicationorganisation.2733

ISSN : $1775-3546$

Éditeur

Presses universitaires de Bordeaux

Édition imprimée

Date de publication : 1 novembre 2002

ISSN : 1168-5549

Référence électronique

Blandine Vanderlinden, «Expliciter l'implicite : à propos des « structures implicites d'organisation » », Communication et organisation [En ligne], 22 | 2002, mis en ligne le 27 mars 2012, consulté le 30 avril 2019. URL : http://journals.openedition.org/communicationorganisation/2733 ; DOI : 10.4000/ communicationorganisation.2733

Ce document a été généré automatiquement le 30 avril 2019.

(c) Presses universitaires de Bordeaux 


\title{
Expliciter l'implicite : à propos des "structures implicites d'organisation »
}

\author{
Blandine Vanderlinden
}

1 La contribution de cet article au questionnement sur les fondements d'une approche interculturelle est sans doute modeste par rapport à l'ambition du projet éditorial. Elle se situe au niveau de la réflexion méthodologique.

2 Nous avons voulu préciser la place faite aux facteurs culturels dans le fonctionnement des organisations et dans le management des entreprises. Dans un premier temps, nous avons commencé par interroger les auteurs contemporains qui ont proposé des outils d'analyse des organisations. Dans un deuxième temps, nous avons cherché à mesurer la pertinence de certains de ces outils en les appliquant à des situations rencontrées lors d'une intervention en entreprise: il s'agit de ceux qui, à travers le prisme de quelques dimensions culturelles, voudraient déterminer les «structures implicites des organisations".

3 La conclusion peut paraître paradoxale. D'une part, elle relève la difficulté d'introduire la différence culturelle dans les schémas d'analyse. D'autre part elle souligne le danger d'une approche qui, en privilégiant les dimensions culturelles, pourrait faire l'impasse sur les autres rapports sociaux en action dans l'entreprise. D'une manière générale, elle indique l'ampleur du chantier qui reste ouvert pour que l'entreprise arrive à reconnaître l'existence de cultures différentes et à redéfinir son rapport à l'autre.

\section{Cultures et organisation : ce qu'on en dit}

Inscrite dans le mouvement de globalisation et soumise à des interférences culturelles multiples, l'entreprise apparaît comme un lieu de tension entre diversité et uniformité. Dès qu'il s'agit d'analyser ces tensions, les positions divergent. Nous aurons à faire la part entre les discours explicites de ceux qui s'instaurent dépositaires d'une école peu encline à remettre en cause un schéma établi et d'autres plus ouverts à la prise en compte des éléments culturels; nous devrons également être attentifs au contenu implicite des 
composantes des organisations qui intègrent nombreux facteurs culturels. Naviguer entre ces courants de pensée c'est être constamment attentif à éviter les deux écueils majeurs que sont la myopie qui dénierait tout rôle aux spécificités culturelles et l'illusion du « tout culturel » qui privilégierait cet élément aux dépens des autres rapports sociaux en œuvre dans les organisations.

\section{Les discours explicites}

$5 \quad$ Il n'est pas facile de repérer clairement la place accordée aux éléments culturels dans la théorie des organisations Cela tient sans doute aux rapports ambigus que certains sociologues ont longtemps entretenus avec la culture. Comme d'ailleurs beaucoup de managers, ils ont eu quelques difficultés à admettre que des facteurs culturels, surtout s'ils venaient de l'extérieur, puissent influencer les organisations. Rien d'étonnant dès lors à ce que les discours officiels se soient longtemps montrés réticents.

6 Gardiens farouches de leur pré carré, certains sociologues ont cru devoir dénier aux éléments culturels toute valeur explicative dans l'analyse des organisations; ils craignaient sans doute que la prise en compte de ces éléments ne vienne minimiser l'influence des rapports sociaux. C'est tout un courant de la sociologie française qui. partent d'une vision réductrice de la culture, s'est opposé à ce qu'ils appelaient «le culturalisme » (Maurice. Sellier. Sylvestre, 1992). Dans la même foulée, assimilant presque G. Hofstede et Ph. d'Iribarne c'est Kriedberg qui après avoir admis avec Crozier que " l'analyse culturelle peut être considérée comme l'autre face de l'analyse stratégique » (Crozier, Friedberg, 1977. p. 190) refusait le prétendu déterminisme de toute approche culturelle (Friedberg. 1997). Enfin Mintzberg lui-même porte sur les rapports entre la culture et les organisations des jugements ambigus : tantôt, il écrit que «l'organisation n'est pas juste une structure, pas même simplement un système de pouvoir: elle est culture (...) c'est une façon de vivre » (Mintzberg, 1990. p. 383) : tantôt, il prétend que la culture, n'étant pas quantifiable ne saurait être prise en compte dans la planification stratégique (Mintzberg. 1994. p. 158-159).

7 Pareil « déni de la différence culturelle » se retrouve dans les théories du management où les variables culturelles ont souvent été écartées au profit des aspects techniques, commerciaux et financiers des activités internationales (Chevrier. 2000, p. 148). Face à la globalisation, deux tendances opposées ont pu être observées: l'une, celle de la convergence, qui voudrait minimiser les différences et tendrait à imposer une stratégie globale qui les transcende ; l'autre, celle de la divergence, qui s'efforcerait de tirer parti des différences et tendrait à développer des formes de management plus adaptées au contexte culturel des pays ou des régions où il s'exerce (Dupriez. Simons. 2002. chap. 4, p. 105). Très longtemps, ce sont les thèses de la convergence qui ont dominé les stratégies des entreprises multinationales.

8 Le chœur n'est cependant pas unanime. Ph. d'Iribarne a attiré l'attention sur les "évidences fondatrices qui donnent sens aux conduites» (d'Iribarne. 1986). R. Sainsaulieu a indiqué comment différentes références culturelles peuvent agir dans l'entreprise (Sainsaulieu. 1987). Delavallée a bien montré qu'une incohérence entre la culture et certaines caractéristiques de l'organisation se traduit par une inadéquation entre les objectifs « affichés » et les objectifs « pratiqués » (Delavallée. 1994, 1996). Crozier lui-même, rappelle que les règles du jeu social sont «à un certain degré des phénomènes de culture » (Crozier. 1989. p. 71). 


\section{Les contenus implicites : la culture dans la théorie des organisations}

9 Bien que la culture n'apparaisse pas comme une variable clé dans la théorie des organisations, elle est cependant présente, parfois explicitement et parfois implicitement, dans certaines variables retenues ; parfois elle est totalement absente.

10 Certains mécanismes de coordination se réfèrent explicitement à un système de valeurs. Les variables politiques, qui traitent des buts de l'organisation et du pouvoir des acteurs, y font implicitement référence. Par contre, les variables de contexte, telles qu'elles sont exprimées, les ignorent totalement.

\section{La standardisation des normes et la mobilisation idéologique}

11 La standardisation des normes, parfois appelée «idéologie organisationnelle » consisterait selon Minfzberg en « un système de croyances et de valeurs à propos de l'organisation, auquel tous les membres de l'organisation adhèrent ; c'est un système qui est différent de celui d'autres organisations » (Mintzberg, 1986, p. 222). Cette idéologie a une histoire qui est liée à celle de l'organisation. «Une idéologie prend racine, dès lors qu'un groupe de personnes se constitue autour d'un chef pour créer une organisation, et que naît le sentiment d'une mission à accomplir. L'idéologie, ensuite, en créant des traditions, se développe à travers le temps. Enfin, une idéologie existante s'affermit avec la venue de nouveaux membres qui s'identifient à l'organisation et à son système de croyances » (Mintzberg, 1986, p. 222).

milisation idéologique » qui jouerait comme mécanisme de coordination au niveau des différentes unités, s'inscrit dans le prolongement de la standardisation des normes. Elle consiste à proposer un corps de valeurs communes, censées inspirer l'activité de tous les départements de façon à réduire les divergences qui pourraient apparaître entre les objectifs de chacun d'entre eux.

13 Selon Nizet et Pichault, la standardisation des normes ne peut être considérée comme mécanisme de coordination que si « le contrôle du travail s'effectue essentiellement par le biais d'une idéologie qui tente d'obtenir l'adhésion de chaque travailleur aux valeurs organisationnelles et de gagner ainsi son implication dans la vie de l'entreprise » (Nizet, Pichault, 1995, p. 40). Le même raisonnement peut être tenu pour la mobilisation idéologique.

On le voit, la référence à un certain nombre de valeurs partagées est bien présente dans les mécanismes de coordination, mais nous restons ici au niveau de la culture organisationnelle sans nécessairement prendre en compte les racines culturelles des membres de l'organisation.

\section{Les buts de l'organisation}

H. A. Simon l'a rappelé, tout acte de management implique un choix de valeurs concernant l'homme et la société, et les objectifs de l'entreprise expriment les valeurs qui seront privilégiées dans la décision. Dès lors, tout ce qui se réfère aux buts, qu'ils soient de mission ou de système, est porteur d'un poids culturel. Aucune mention n'y est faite chez Mintzberg. 
16 Pourtant, l'énoncé même des buts de systèmes (croissance de l'entreprise, plan de carrière, formation,...) évoque déjà les valeurs impliquées par chacun d'eux. Pour ce qui est des buts de mission, on a pu faire explicitement référence à des groupes de valeurs, individualistes ou holistiques. Dès qu'un système de valeurs est en cause, on se trouve sur le terrain de la culture.

\section{Les acteurs et leur pouvoir}

17 L'analyse des relations de pouvoir entre les acteurs fait assez peu appel aux références culturelles.

18 Cependant, dans l'approche de Crozier et Friedberg, l'ouverture aux réalités culturelles est assez franche. Individus ou groupes, avant d'être membres d'une organisation, les acteurs sont membres d'autres groupes sociaux marqués par leur propre culture. Celle-ci n'est pas abandonnée à l'entrée d'une organisation comme on pourrait laisser un vêtement de ville au vestiaire avant de revêtir son bleu de travail. Certaines traditions restent profondément ancrées et peuvent induire des lignes d'action ou des forces de résistance. Cela peut expliquer qu'un individu, tout en restant raisonnable, puisse adopter un comportement qui s'écarte des objectifs de l'organisation.

19 En reconnaissant que les acteurs sont en situation de rationalité limitée, on admet que les individus peuvent adopter un comportement qui n'est pas conforme au rôle que leur attribue l'organisation, que ce comportement peut répondre à d'autres critères que les valeurs admises par l'organisation (Dupriez. Simons. 2002. chap. 3). C'est ouvrir la porte à l'analyse d'autres réalités, dont les réalités culturelles, ce que justifie la phrase déjà citée de Crozier et Friedberg : «l'analyse culturelle peut être considérée comme l'autre face de l'analyse stratégique ».

\section{Les facteurs de contingence}

20 Aucune mention des références culturelles n'est faite dans l'analyse des variables de contexte. Ce silence peut étonner car la réalité à laquelle Mintzberg se réfère est, à l'évidence, porteuse de culture.

21 Même les références qualifiées d'interne, l'âge d'une entreprise, sa taille ou la technologie utilisée, sont loin d'être imperméables aux réalités culturelles. C'est là que Thévenet propose de rechercher ce qu'il appelle les "empreintes" de la culture d'entreprise (Thévenet, 1993).

Dans l'analyse de Mintzberg, la place privilégiée est réservée aux contraintes du marché dont les contours seraient définis en termes exclusivement économiques. Or ces contraintes sont celles qui font intervenir l'environnement dans lequel vit et travaille l'entreprise. Cet environnement est loin d'être exclusivement économique et on découvre aujourd'hui l'importance des éléments culturels dans celui-ci.

23 Cette emprise du culturel implique l'entreprise dans sa totalité. Elle commence par la culture nationale ou par les racines culturelles dans lesquelles toute entreprise est plongée. Elle se prolonge ensuite au sein des organisations par une culture qui lui est propre, la culture organisationnelle. 


\section{G. Hofstede et les structures implicites d'organisation}

D'autres voies ont été ouvertes qui pourraient combler les lacunes de certaines présentations des théories de l'organisation. Ainsi, l'approche de G. Hofstede introduit une dimension culturelle dans l'analyse des organisations.

C'est à partir des éléments rassemblés dans un travail de recherche impressionnant effectué auprès de 116000 cadres d'une entreprise multinationale qu'Hofstede a pu concevoir une approche des organisations qui puisse prendre en compte les composantes culturelles.

Au départ, ses enquêtes ont permis de construire une typologie des cultures basée sur quatre critères: la distance hiérarchique, le contrôle (l'évitement) de l'incertitude, l'individualisme et la masculinité, ou leur contraire. Ces quatre dimensions « constituent un cadre général qui souligne les faits les plus apparents et les plus frappants de la relativité culturelle ;(..) elles décrivent les problèmes de base de l'humanité sur lesquels chaque société doit prendre parti. La variation de chaque pays sur ces dimensions montre la prise de position de chaque société par rapport à ces problèmes ». (Bollinger, Hofstede. 1987. p. 155-156).

Hofstede lui-même souligne certaines limites de cette typologie : «il se peut très bien qu'il existe d'autres dimensions de la culture que l'on n'a pas trouvées dans notre recherche internationale: tout simplement parce que l'on n'a pas posé les questions pertinentes » (Bollinger, Hofstede. 1987, p. 156).

28 Les termes utilisés indiquent bien qu'il s'agit d'une méthode comparative et, pour l'approche approfondie des dimensions d'une culture donnée, celle-ci permet seulement de faire une partie du chemin (Dupriez, Simons, 2002. p. 47-54). Mais ce qui nous intéresse ici c'est l'implication de ces dimensions culturelles dans la structure des organisations.

Hofstede croit pouvoir classer les organisations en partant de deux éléments principaux :

- la centralisation du pouvoir d'une part.

- le degré de standardisation, de spécialisation, de planification et de formalisation des rôles, d'autre part.

Or, ces deux éléments clés correspondent également aux deux dimensions culturelles qu'il a définies précédemment : la distance hiérarchique et le contrôle de l'incertitude.

La distance au pouvoir indique le degré de tolérance d'une société par rapport à l'inégalité du pouvoir dans les institutions. «La hauteur de la distance hiérarchique fournit la réponse au degré de centralisation des décisions qui s'instaure spontanément dans un pays: plus la distance hiérarchique est haute, plus l'on verra apparaître une centralisation du pouvoir. À l'inverse, plus la distance hiérarchique entre les hommes sera courte, plus facilement l'on pourra instaurer une décentralisation des décisions. »

L'évitement (le contrôle) de l'incertitude indique le degré de tolérance pour l'incertitude et l'ambiguïté1. Dans une culture à fort évitement de l'incertitude, on aura tendance à multiplier les règlements et à valoriser le conformisme social : la tendance au consensus y est forte, rendant inacceptables les comportements déviants. Dans une culture plus tolérante à l'égard de l'incertitude, les comportements individuels sont moins contrôlés et il y a plus de place pour les initiatives personnelles.

«Le contrôle de l'incertitude constitue une clé importante pour comprendre les entreprises, car la façon dont elles perçoivent et contrôlent leurs environnements, 
entraîne des conséquences fondamentales pour les différents types de structure. En effet, le contrôle de l'incertitude explique le degré de formalisation des rôles dans une entreprise, c'est-à-dire la description des postes, la standardisation des règles et des procédures, la mise en place d'organigrammes détaillés, etc.

Dans les pays connaissant un fort contrôle de l'incertitude, les organisations se tourneront spontanément vers une structure formalisée, alors que dans les pays avec un faible contrôle de l'incertitude on préfère plutôt les structures informelles, dans lesquelles on négocie au moment approprié ».

valeurs culturelles nationales se caractérisent par un faible degré d'évitement de l'incertitude et une faible distance au pouvoir ; elle n'est ni formalisée ni centralisée. Les relations entre les individus et les procédures de travail sont le plus souvent ouvertes à la négociation et à l'improvisation. Le patron est responsable et les subordonnés reçoivent des objectifs à atteindre.

Graphique 1 - Les structures implicites d'organisation, des entreprises selon Hofstede

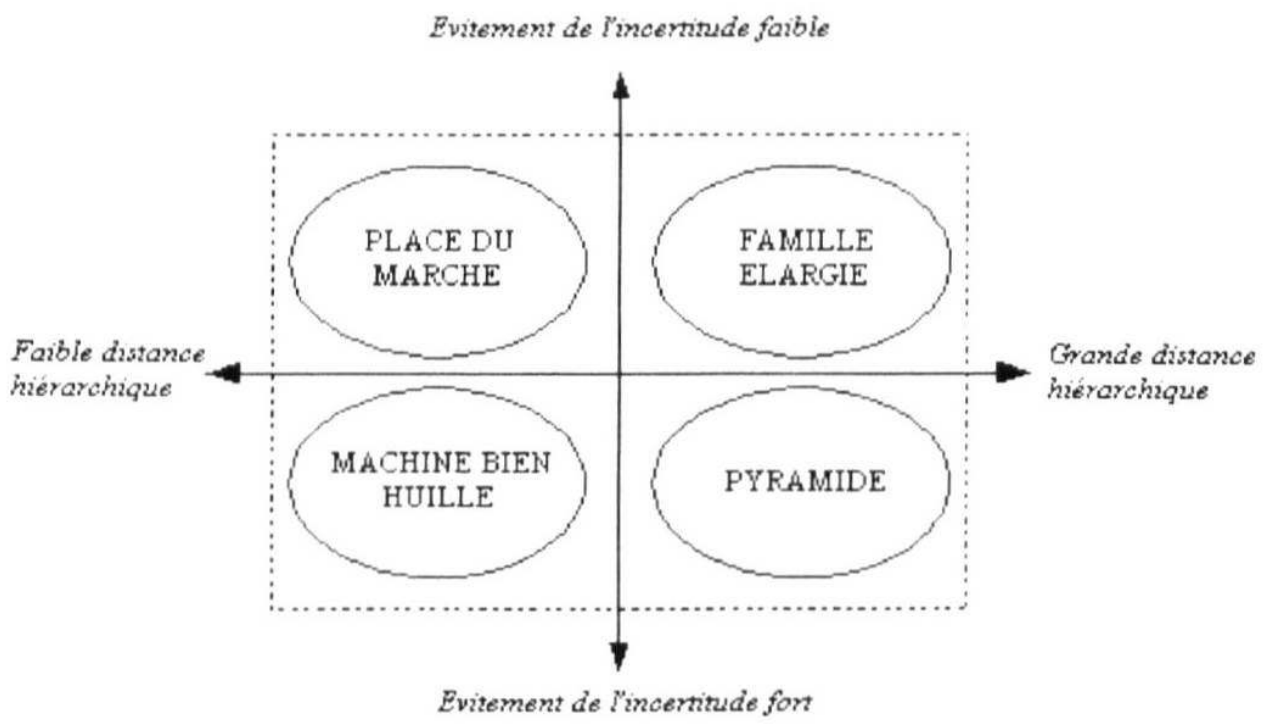
alliée à un fort évitement de l'incertitude. La structure est formalisée mais décentralisée. Là, contrairement aux types précédents, les procédures de travail sont strictement prévues, sauf les relations entre les individus hors de leurs rôles. Le patron est un expert et les subordonnés sont tenus de respecter strictement les règles établies. 

présente dans les pays où les valeurs culturelles nationales se caractérisent par une grande distance hiérarchique et un fort évitement de l'incertitude. La structure est à la fois formalisée et centralisée. Les procédures de travail, aussi bien que les relations entre les individus, sont prévues d'une manière rigide, soit par des règles formelles ou des lois, soit par la coutume et la tradition. Le patron est autoritaire et donne des instructions à ses subordonnés.

Cette analyse ouvre une voie à la prise en compte de l'influence de certains éléments culturels dans les organisations. C'est ce qui en fait tout l'intérêt et c'est ce qu'ont sans doute apprécié les légions de chercheurs et de consultants qui ont pris l'habitude d'aborder les organisations au regard des critères d'Hofstede. On doit cependant se demander si. en privilégiant ainsi les éléments culturels, elle peut rendre compte adéquatement du fonctionnement des organisations.

\section{Les structures implicites en question}

L'application de cette analyse à des situations rencontrées lors d'une intervention en entreprise devrait permettre d'en mesurer la pertinence pour proposer une lecture des situations concrètes. Il convient d'abord d'en préciser les limites théoriques.

\section{Les limites théoriques}

42 Au niveau de l'analyse des organisations, cette approche ignore deux dimensions majeures $d u$ fonctionnement de celles-ci: D'une part, l'approche de Hofstede fait l'impasse sur les mécanismes de coordination entre les acteurs et sur les mécanismes de liaison entre les unités qui composent l'organisation. D'autre part, la dimension qualifiée de distance au pouvoir n'établit pas de distinction entre la notion de hiérarchie et celle de pouvoir (d'Iribarne. 1997. p. 30-47). Elle semble relever plus du mode de distribution du pouvoir que des jeux de pouvoirs. Or, ni sur le plan théorique ni sur le terrain concret des organisations, on ne peut ignorer la place occupée par les jeux de pouvoir (Vanderlinden. 2003).

Même en adoptant le raisonnement d'Hofstede, il faut encore préciser les conditions de son application aux situations rencontrées. Les dimensions retenues, à savoir la distance hiérarchique et l'évitement de l'incertitude, ont d'abord permis à Hofstede de caractériser les cultures nationales. Ce n'est qu'indirectement qu'il envisage que des types d'organisation correspondent à ces cultures. Cela suppose que deux conditions au moins soient réalisées :

44 1) que les critères retenus et leur application nous donnent une approche pertinente de la culture nationale, et.

45 2) que le type d'organisation de chaque entreprise soit conforme au modèle national ainsi exprimé.

\section{L'épreuve du terrain}

L'entreprise étudiée est un groupe international spécialisé à l'origine dans la fabrication de matériaux de couverture des bâtiments, que nous avons choisi d'appeler Couvrex.

Communication et organisation, 22 | 2012 
Plusieurs niveaux différents ont été retenus pour procéder à cette vérification : tout d'abord celui du Groupe, établi en Belgique, et celui de sa filiale chilienne que nous avons appelée Materiales qui ont tous deux fait l'objet d'une étude approfondie ; celui ensuite de deux filiales, l'une française, l'autre allemande, qui n'ont été abordées que de manière indirecte.

Le développement international de ce groupe a surtout reposé sur l'acquisition de sociétés et la diversification des produits. Devenue plus complexe, la structure a toléré des différences importantes d'une zone géographique à l'autre, voire d'un pays ou d'une implantation à l'autre. En raison des points forts ou des points faibles de chaque entité, des fonctions comme le management général, certains aspects techniques ou la gestion financière ont parfois été confiés à des cadres expatriés, porteurs des références du groupe. Pour chaque niveau abordé, les logiques d'organisation peuvent se rapprocher ou se différencier. Certaines valeurs sont partagées par l'ensemble du groupe, d'autres sont spécifiques à telle ou telle filiale: d'autres encore sont propres à un pays ou à une implantation.

Nous commencerons par un aperçu général qui reprendra les critères d'Hofstede. Nous procéderons ensuite à l'analyse des entreprises que nous connaissons dans chacun des pays concernés et nous essaierons de les situer dans la typologie proposée par Hofstede.

\section{Un aperçu général}

Dans le tableau 1. nous avons repris les critères d'Hofstede pour les quatre pays concernés par notre analyse. Afin de mieux percevoir la portée de cette classification, nous avons indiqué entre parenthèses les pays occupant des positions extrêmes.

Tableau1 - Les deux dimensions d'Hofstede dans les pays concernés, d'après Hofstede (1994, p. 26,113$)$

\begin{tabular}{|c|c|c|c|c|c|}
\hline \multicolumn{3}{|c|}{ Distance hiérarchiq ue } & \multicolumn{3}{|c|}{ Évitement de l'incertitude } \\
\hline Rang & Pays & Indice & Rang & Pays & Indice \\
\hline (1) & Malaisie & $(104)$ & (1) & Grèce & $(112)$ \\
\hline 16 & France & 68 & 5 & Belgque & 94 \\
\hline 20 & Belgque & 65 & 10 & France & 86 \\
\hline 24 & Crili & 63 & 11 & Chili & 86 \\
\hline 43 & Allemagne (W) & 35 & 29 & Allemagne (W) & 65 \\
\hline (53) & Autriche & (11) & $(53)$ & Singapour & (8) \\
\hline
\end{tabular}

d'après H ofstede (1994, p. 26, 113)

Par rapport à ces deux critères, la France, la Belgique et le Chili apparaissent dans des positions très proches. L'évitement de l'incertitude y serait assez élevé, davantage en 
Belgique qu'en France et au Chili. Dans ces trois pays également, la distance hiérarchique serait assez élevée. Ce qu'Hofstede explique par une appartenance commune à la culture latine (Bollinger. Hofstede, 1987. p. 90).

51 L'Allemagne, par contre ferait en quelque sorte bande à part avec un indice plus faible tant pour l'évitement de l'incertitude que pour la distance hiérarchique

Ces données peuvent être reportées sur la carte des structures implicites tracée par Hofstede qui suggère que les pays mettent en place des structures d'organisation correspondant à leur « mentalité » telle que l'expriment les critères relevés.

Dans ce cas comme l'indique le graphique 2, les entreprises du Chili, de la France et de la Belgique seraient du type pyramide. L'Allemagne, par contre, se trouverait dans une position intermédiaire entre la machine bien huilée et la place du marché.

Ces résultats nous interpellent.

Par rapport à la première condition évoquée au début de cette section nous sommes d'emblée surprise du positionnement relatif des différents pays dans ce classement. Le degré d'évitement de l'incertitude de la Belgique, comparativement au Chili et à la France, y serait plus élevé ; il serait le même en France et au Chili.

\section{Graphiques 2 - Les structures implicites d'organisation dans quelques pays d'implantation de Couvrex}

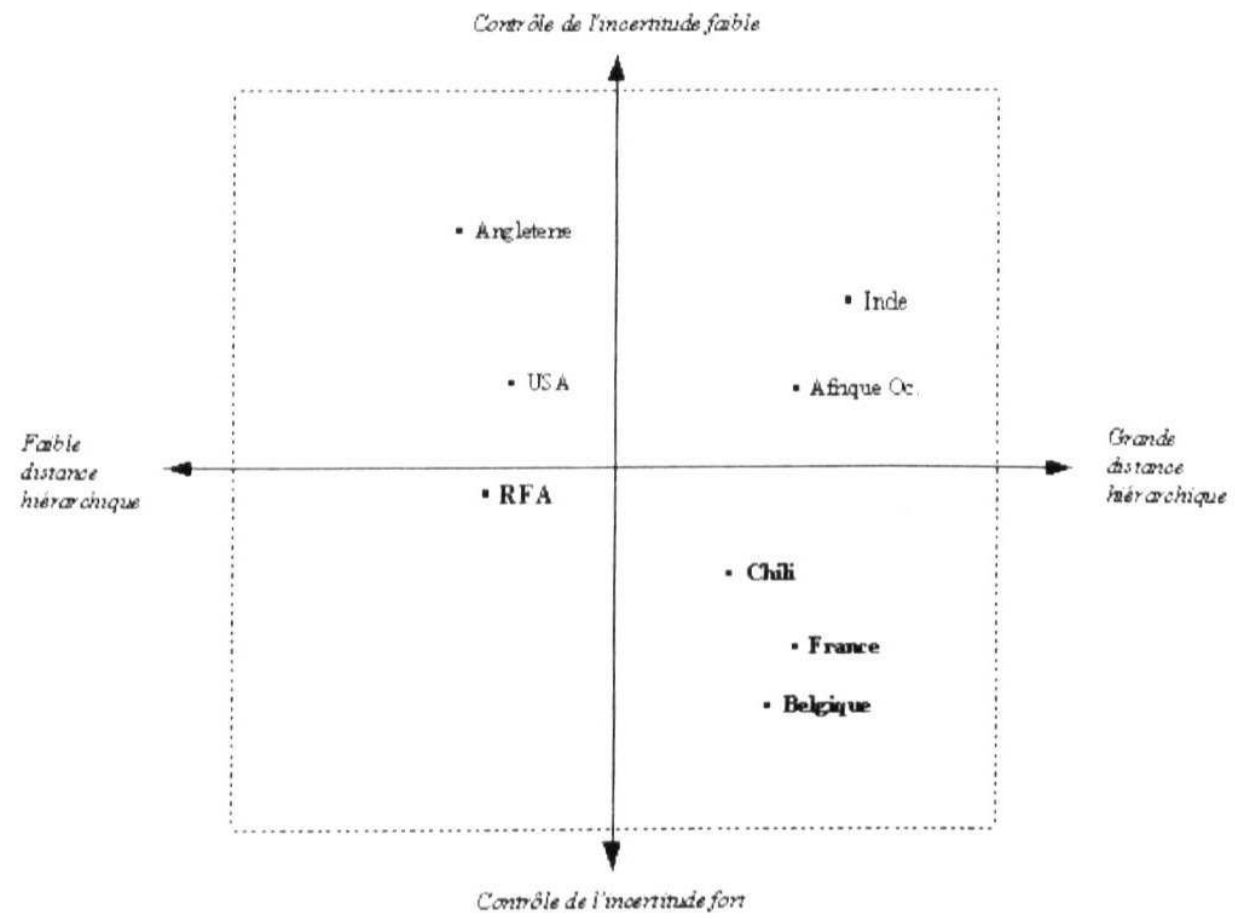

d'apres G Hofstede (Bollinger, Hof stede, 1987, p 229)

d'après G Hofstede (Bollinger, Hofstede, 1987, p. 229)

Rappelons que pour Hofstede, cette « dimension culturelle mesure le degré de tolérance qu'une culture peut accepter face à l'inquiétude provoquée par des évènements futurs »; un contrôle fort signifie que la tolérance est faible (Bollinger, Hofstede, 1987, p. 103). Il ajoute que les pays à fort contrôle de l'incertitude pourraient être considérés comme des pays fermés. Ce positionnement ne nous paraît pas correspondre aux valeurs vécues par 
les sociétés concernées et nous devons donc nous interroger sur la réalisation de la première condition.

Quant à la seconde condition, il faut rappeler les réserves d'ordre théorique déjà évoquées : le critère de la distance hiérarchique tel qu'il est formulé par Hofstede n'a pas pris en compte l'existence des jeux de pouvoir ni celle des mécanismes de coordination intervenant dans les organisations. Ces deux éléments, fondamentaux dans les approches conjuguées de Mintzberg et de Crozier. ne peuvent être négligés dans l'approche de la réalité concrète d'une organisation.

Pour tenter d'élucider ces questions et sans vouloir contester le fruit d'une étude sérieuse et reconnue, nous allons maintenant examiner chaque groupe ou sous-groupe rencontré en nous demandant où il se situe par rapport à la typologie proposée par Hofstede.

La démarche adoptée par Hofstede pour caractériser les types d'organisation est déductive en ce sens qu'elle déduit les structures implicites de l'organisation des caractéristiques culturelles attribuées à un pays. À l'inverse, la nôtre partira de l'observation d'une organisation concrète que nous tâcherons de rattacher à l'un ou l'autre type d'organisation envisagé par le modèle.

\section{Une analyse groupe/pays}

60 Nous ne disposons pas de beaucoup d'éléments pour décrire l'organisation des groupes français et allemand étant donné qu'ils n'ont pas fait l'objet d'une attention particulière dans le cadre de notre étude. Cependant certains témoignages de cadres interviewés nous permettent d'en percevoir quelques traits majeurs.

\section{Le Groupe belge}

61 La distance hiérarchique est faible.

62 La ligne hiérarchique est aplatie, les relations interpersonnelles sont privilégiées et la communication est informelle. Il n'y a pas de symboles de statut et même l'Administrateur délégué est low profile. Le pouvoir est occulté, et du sommet au simple employé, on se parle avec cordialité, respect et sympathie.

L'organisation est décentralisée ce qui reflète une confiance accordée aux membres. Il est vrai qu'on utilise le vouvoiement, on prend rendez-vous, on se fait annoncer; mais ce sont plutôt des signes de respect de la liberté d'autrui. des manifestations d'une rigueur et d'une organisation dans le travail.

Le degré d'évitement de l'incertitude est faible.

L'organisation a toujours privilégié le pragmatisme et la souplesse, les directives sont générales et l'initiative est laissée aux individus, les règles peuvent être transgressées pour des raisons pragmatiques et l'attention est portée aux résultats, quels que soient les moyens utilisés pour y parvenir (dans les limites de certaines directives telles que la qualité, l'environnement,...).

La délégation à des subordonnés peut être complète, on tolère l'ambiguïté dans la perception des autres, la concurrence entre les employés est loyale, des étrangers peuvent exercer des fonctions dirigeantes et beaucoup de personnes voyagent fréquemment ou ont séjourné à l'étranger. 
67 Par rapport aux axes de Hofstede, nous pouvons dire que le Groupe belge est une organisation de type place du marché ; elle est ni formalisée ni centralisée.

\section{Le sous-groupe français :}

La distance hiérarchique est forte.

Les Français sont perçus comme très hiérarchiques, avec des jeux de pouvoir internes nombreux. Les décisions sont centralisées et des règles formelles sont imposées. Le pouvoir est clairement exprimé et toute autre autorité les dérange. Un sentiment de supériorité par rapport au groupe belge est manifesté et les membres du sous-groupe français semblent avoir une faible ouverture à la différence.

Le degré d'évitement de l'incertitude est fort.

Les structures hiérarchiques sont claires et respectées. Les règles et procédures sont nécessaires pour motiver le personnel et quand on délègue à des subordonnés on doit contrôler leurs initiatives. Le mode de raisonnement est absolutiste et on ne tolère pas d'ambiguïté dans la perception d'autrui (il est bon ou mauvais). Par rapport aux axes de Hofstede, le sous-groupe français serait de type pyramide ; les structures sont formalisées et centralisées

\section{Le sous-groupe allemand}

La distance hiérarchique est faible.

Le sous-groupe allemand est perçu comme un bloc fermé sans ouverture à un autre modèle de management ou toute forme d'interférence du groupe belge et il ne peut souffrir de perdre sa suprématie.

Les autres membres du Groupe les perçoivent comme étant très hiérarchiques, avec des supérieurs distants et autoritaires. Mais cette distance relève davantage de l'exercice du pouvoir par rapport au Groupe belge qu'à une distance hiérarchique interne. En effet les décisions sont généralement décentralisées mais uniquement à l'intérieur de leur environnement.

Le degré d'évitement de l'incertitude est fort.

Le sous-groupe allemand est très procédurier et les règles formelles établies sont généralement bien respectées. Les champs d'action sont strictement limités, chaque personne réalisant un travail rigoureux et de qualité mais sans perspective globale. Les compétences sont limitées aux responsabilités et il y a peu d'intérêt de s'ouvrir à d'autres réalités.

7 Par rapport aux axes de Hofstede. le sous-groupe allemand serait de type machine bien huilée : il a une structure décentralisée et formalisée.

\section{La filiale Materiales}

9 Les caractéristiques de la culture latine relevées par Hofstede sont présentes dans l'organisation Materiales.

Il s'agit d'une forte distance au pouvoir, lorsque l'on considère le leader de l'entreprise comme une sorte de « dieu » que seuls ses collaborateurs directs peuvent approcher. En effet, le Directeur de la holding est au sommet et relativement inaccessible. La ligne 
hiérarchique dans les filiales est longue et les informations doivent être transmises d'échelon en échelon.

81 L'évitement de l'incertitude est également fort dans la culture latine ce qui se traduit dans les filiales par des règles de conduite que tout employé doit strictement respecter. Une première lecture de l'organisation pourrait conduire à considérer que Materiales est de type pyramide.

Cependant, d'autres facteurs entrent en ligne de compte qui modifient l'appréciation de ces caractéristiques : il s'agit du pouvoir et du charisme du leader de cette organisation.

En effet, le leader a mis en place un mode de fonctionnement particulier qui correspond à ses valeurs et à ses principes de gestion. Grâce à sa forte personnalité et au pouvoir clairement exprimé qu'il représente, les valeurs qui sont les siennes sont partagées par tous les membres de l'organisation.

85 Si l'on considère la distance hiérarchique, elle est à la fois forte et faible. Elle est forte par la force du pouvoir du leader qui centralise toutes les décisions. Elle est faible par le mécanisme de coordination fondé sur les relations interpersonnelles. Le mode de gestion défini au sommet doit être répercuté en cascade à tous les niveaux de la ligne, chaque responsable ayant la responsabilité de le transmettre à ses subordonnés. Les responsables de filiales travaillent en parfaite entente et un esprit de collaboration et d'entraide est présent. Le travail en équipes est un principe de base et les propositions d'initiatives sont prises en compte. Le personnel travaille pour le groupe et s'assimile à celui-ci. Materiales est une grande famille et le travail repose beaucoup sur les relations affectives.

Si l'on considère le degré d'évitement de l'incertitude, il est également relativement faible. Une des caractéristiques majeures de Materiales est cette flexibilité attendue du personnel. Le passage d'une fonction à une autre (de financière à marketing par exemple), d'une entreprise à une autre, d'un pays à un autre, d'un niveau hiérarchique à un autre (et même de descendre de niveau) sont des signes d'une faible résistance au changement.

Cette flexibilité se manifeste également par le refus d'avoir un département des ressources humaines au niveau de la holding et de prévoir toute forme de gestion de carrière. Le refus de procéder à des évaluations à cause des conséquences psychologiques qu'elles engendrent correspond à la non acceptation de rigidifier la perception qu'un supérieur a de son subordonné. D'autre part, le système de gestion des ressources humaines Groupe n'est pas apprécié parce qu'il est perçu comme un système administratif et formalisé. La difficulté d'introduire un système ISO est un autre exemple du refus de mettre en place un système reposant sur des procédures.

Ces observations permettent de dire que l'organisation Materiales associe le type famille élargie et le type place du marché.

Nos propres observations sur deux entreprises étudiées en profondeur et sur deux sousgroupes nationaux que nous ne connaissons qu'indirectement nous conduisent à des résultats différents de ceux que suggère l'étude de Hofstede. 
Tableau 2 - Structures d'organisation implicites et effectives dans quatre pays d'implantation de Couvrex

\begin{tabular}{|c|c|c|c|}
\hline \multicolumn{2}{|c|}{$\begin{array}{l}\text { Structures imp licites d'organisation } \\
\text { selon Hofsted e }\end{array}$} & \multicolumn{2}{|c|}{$\begin{array}{l}\text { Structures d'organisation effectives des } \\
\text { entrep rises du Groupe Couv rex }\end{array}$} \\
\hline Belgque & Pyramide & Le siege de Courrex & Place du marché \\
\hline Chli & Pyramide & Materiales & $\begin{array}{l}\text { Famille élarge - place du } \\
\text { marché }\end{array}$ \\
\hline France & Pyramide & Sous-groupe français & Pyramide \\
\hline Allemagne & $\begin{array}{l}\text { Machine bien huslee - } \\
\text { place du marché }\end{array}$ & Sous-groupe allem and & Machine bien huslee \\
\hline
\end{tabular}
ignoré cette dimension.
Pour les sous-groupes français et allemands, les résultats semblent concordants. Ils divergent radicalement pour le groupe Couvrex et pour la filiale Materiales.

En ce qui concerne le Groupe Couvrex, une première raison de divergence pourrait tenir au fait que les résultats avancés pour les deux dimensions retenues par Hofstede ne semblent pas rendre parfaitement compte des valeurs partagées par la société belge.

Mais dans les deux cas les lacunes de l'analyse théorique des organisations qui se trouve à la base du raisonnement d'Hofstede paraissent être en cause. En effet, dans ce cas, les différences observées semblent principalement liées au choix des mécanismes de coordination et aux jeux de pouvoir en action dans les entreprises concernées.

\section{Méthodologie et ouverture à l'altérité}

La prise en compte des éléments culturels dans l'analyse des organisations comme dans les théories du management est loin d'être évidente. Le plus souvent, il faut recourir à une lecture attentive des réalités observées pour alimenter un cadre conceptuel qui avait

Par ailleurs Hofstede a, en quelque sorte, popularisé l'analyse des éléments culturels et en a déduit une typologie des organisations. Nous avons été amenée à en indiquer les limites : la culture n'éteint pas les rapports sociaux en œuvre dans les organisations.

Les limites de l'analyse théorique n'enlèvent rien à la richesse et à la complexité de la réalité sociale et la difficulté de la prise en compte des éléments culturels ne rend pas inutiles les tentatives, conceptuelles ou appliquées, d'y parvenir. Même si l'univers de la diversité culturelle est complexe et parfois insaisissable, les entreprises ne peuvent plus ignorer les différences culturelles.

96 C'est tout l'enjeu du management interculturel développé ailleurs (Dupriez, Simons, 2002, chap. 4 ; Dupriez, Vanderlinden, Soumah-Mis, 2002). Au niveau de l'entreprise, comme au 
niveau de la société, il exprime la capacité à reconnaître l'autre dans ses valeurs propres et à intégrer la différence dans son propre système de pensée et d'action (Pierre, 2001, p. 121). Supposant une capacité à se remettre en cause, il apparaît comme une forme de résistance à toute tendance à l'uniformité du management et de la culture (Dupriez, 2002).

\section{BIBLIOGRAPHIE}

BOLLINGER D., HOFSTEDE G., Les différences culturelles dans le management : comment chaque pays gère-t-il ses hommes? Éditions d'Organisation : Paris, 1987.

CHEVRIER S., Le management des équipes interculturelles. Paris : PUF, 2000.

CROZIER M., FRIEDBERG E., L'acteur et le système. Paris : Seuil. 1977.

CROZIER M.. L'entreprise à l'écoute, Paris : Seuil, 1989.

d'IRIBARNE P., HENRY A., SEGAL J-P, CHEVRIER S. et GLOBOKAR T., Cultures et mondialisation. Gérer par-delà les frontières. Paris : Seuil 1998.

DELAVALLEE E., Culture et décision, le cas d'une entreprise de haute technologie. Paris, I.A.E., thèse. 1994, cité dans DELAVALLEE E., « Pour ne plus gérer sans la culture », in Revue française de gestion, $1996, n^{\circ} 110$, pp. 5-16.

DUPRIEZ P., SIMONS S.. La résistance culturelle : fondements, applications et implications $d u$ management interculturel. Bruxelles : De Boeck, deuxième édition, 2002.

DUPRIEZ P., VANDERLINDEN B. et SOUMAH-MIS O., « Balises pour le management interculturel ». La semaine internationale. Nancy : ICN -Université Nancy 2. 2002-2003.

DUPRIEZ P., « De la souveraineté confisquée à la résistance culturelle ». Colloque CEDIMES, Souveraineté et management dans un contexte de mondialisation. Université de Wroclaw : mai 2002. FRIEDBERG E., Le pouvoir et la règle : dynamiques de l'action organisée. Paris : Seuil. 1997.

HOFSTEDE G. et al., « Measuring Organizational Cultures : a Qualitative and Quantitative Study Accross Twenty Cases. » in : Administrative Science Quarterly. 1990. n². pp. 286-316.

HOFSTEDE G., Cultures and Organizations : Software of the Mind. London : Harper Collins Publishers, 1994.

MAURICE M., SELLIER F, SYLVESTRE J.-J., « Analyse sociétale et cultures nationales : réponse à Philippe d'Iribarne », in Revue française de sociologie. 1992, XXXIII, pp. 75-76

MINTZBERG H., Le pouvoir dans les organisations. Paris : Ed. d'Organisation, 1986

MINTZBERG H., Le management. Voyage au centre des organisations. Paris : Ed. d'Organisation, 1990.

MINTZBERG H., Grandeur et décadence de la planification stratégique. Paris : Dunod, 1994.

MORIN P., DELA VALLEE E., Le manager à l'écoute du sociologue. Paris : Éditions d'Organisation 2001.

NIZET J., PICHAULT F., Comprendre les organisations. Mintzberg à l'épreuve des faits. Paris :

Gaétan Morin, 1995. 
NIZET J., PICHAULT F., Introduction à la théorie des configurations. Du « one best way » à la diversité organisationnelle. Bruxelles : De Boeck, 2001.

PIERRE P., «Éléments pour une réflexion critique sur le management interculturel », Sociologies pratiques, $\mathrm{n}^{\circ} 5$, décembre 2001

SAINSAULIEU R., Sociologie de l'organisation et de l'entreprise. Paris : Dalloz, 1987.

SAINSAULIEU R., L'identité au travail. Paris : Fondation Nationale des Sciences politiques. 1988.

THEVENET M., La culture d'entreprise. Paris : P.U.F., 1993.

VANDERLINDEN B., « Le papillon des Andes, Éléments d'une analyse contextuelle des cultures organisationnelles ». Sociologies pratiques, (à paraître) 2003.

\section{NOTES}

1. Dans les éditions en langue anglaise (Hofstede. 1984). le terme utilisé est «Uncertainty Avoidance » qui pourrait être traduit par « Evitement de l'incertitude » qui évoque une réaction psychologique. Une édition en langue française (Bollinger. Hofstede. 1987) emploie l'expression «Contrôle de l'incertitude » qui nous semble avoir une connotation différente. Nous emploierons l'expression "contrôle » chaque fois que nous nous référerons plus ou moins explicitement à l'édition en langue française et « évitement » dans les autres cas.

\section{RÉSUMÉS}

Blandine Vanderlinden tente de préciser la place faite aux facteurs culturels dans le fonctionnement des organisations et le management des entreprises. Elle interroge des auteurs qui ont proposé des outils d'analyse des organisations; elle cherche ensuite à mesurer la pertinence de certains outils en les appliquant à des situations rencontrées : ceux qui, à travers quelques dimensions culturelles, voudraient déterminer les «structures implicites des organisations ». La conclusion montre la difficulté d'introduire la différence culturelle dans les schémas d'analyse et souligne le danger d'une approche qui, en privilégiant les dimensions culturelles, pourrait faire l'impasse sur les autres rapports sociaux en action dans l'entreprise. Elle indique le chemin à parcourir pour reconnaitre l'existence de cultures différentes et redéfinir son rapport à l'autre.

Blandine Vanderlinden wishes to specify the place of cultural factors in the management of the organisations. She begins by consulting other researchers who have proposed analytical tools applicable to organisations. Next she attempts to measure the importance of some tools by applying them to real-life situations. These tools, through the prism of some cultural dimensions, seem to determine the "implicit structure of the organisations". The conclusion may appear paradoxical. On the one hand she indicates the difficulty of making the cultural differences as a subject of the analysis. On the other hand, she sees as dangerous to limit the analysis of the organisation to the cultural dimension without taking under consideration social aspects. She 
emphasizes that the company has to recognize the existence of various cultures and to redefine its attitude to others

INDEX

Mots-clés : Culture organisationnelle, facteurs culturels, organisations, structure implicite

\section{AUTEUR}

\section{BLANDINE VANDERLINDEN}

Blandine Vanderlinden, Chef de travaux à l'ICHEC. Bruxelles et Secrétaire académique et scientifique du MIME, www.mimc.ichec.be Ses recherches sont orientées dans le domaine de la culture organisationnelle : blandine.vanderlinden@mime.ichec.be 